\title{
New analytical solutions for quark stars with charged anisotropic matter
}

\author{
Manuel Malaver \\ Universidad Marítima del Caribe, Departamento de Ciencias Básicas, Catia la Mar, Venezuela \\ Email address: \\ mmf.umc@gmail.com
}

\section{To cite this article:}

Manuel Malaver. New Analytical Solutions for Quark Stars with Charged Anisotropic Matter. International Journal of Astrophysics and Space Science. Special Issue: Compact Objects in General Relativity. Vol. 3, No. 1-1, 2015, pp. 6-12.

doi: $10.11648 /$ j.ijass.s.2015030101.12

\begin{abstract}
In this paper, we found new exact solutions to the Einstein- Maxwell system of equations within the framework of MIT Bag Model considering a particular form for the measure of anisotropy and a gravitational potential which depends on an adjustable parameter $\alpha$. The first class of solutions has a singularity in the center. The second class of solutions is regular in the stellar interior. Variables as the energy density, radial pressure, tangential pressure, electric field intensity and the metric functions are written in terms of elementary and polinominal functions. We show that the form chosen for the gravitational potential and the anisotropy allows obtain physically acceptable solutions with any value of the adjustable parameter.
\end{abstract}

Keywords: Gravitational Potential, Adjustable Parameter, Einstein-Maxwell System, MIT Bag Model, Energy Density, Measure of Anisotropy

\section{Introduction}

One of the fundamental problems in the general theory of relativity is finding exact solutions of the Einstein field equations [1,2]. Some solutions found fundamental applications in astrophysics, cosmology and more recently in the developments inspired by string theory [2]. Different mathematical formulations that allow to solve Einstein's field equations have been used to describe the behavior of objects submitted to strong gravitational fields known as neutron stars, quasars and white dwarfs $[3,4,5]$.

From the development of Einstein's theory of general relativity, the description of compact objects has been a central issue in relativistic astrophysics in the last few decades [2,6]. Recent experimental observations in binary pulsars [6] suggest that could be quark stars. The existence of quark stars in hydrostatic equilibrium was first by Itoh [7] in a seminal treatment. Recently, the study of strange stars consisting of quark matter has stimulated much interest since could represent the most energetically favourable state of baryon matter.

In the construction of the first theoretical models of relativistic stars are important the works of Schwarzschild [8], Tolman [9] and Oppenheimer and Volkoff [10]. Schwarzschild [8] found analytical solutions that allowed describing a star with uniform density, Tolman [7] developed a method to find solutions of static spheres of fluid and Oppenheimer and Volkoff [8] used Tolman's solutions to study the gravitational balance of neutron stars. It is important to mention Chandrasekhar's contributions [11] in the model production of white dwarfs in presence of relativistic effects and the works of Baade and Zwicky [12] who propose the concept of neutron stars and identify an astronomic dense objects known as supernovas.

The physics of ultrahigh densities is not well understood and many of the strange stars studies have been performed within the framework of the MIT bag model [13]. In this model, the strange matter equation of state has a simple linear form given by $p=\frac{1}{3}(\rho-4 B)$ where $\rho$ is the energy density, $\mathrm{p}$ is the isotropic pressure and B is the bag constant. However, theoretical works of realistic stellar models [14-17] it has been suggested that superdense matter may be anisotropic, at least in some density ranges. The existence of anisotropy within a star can be explained by the presence of a solid core, phase transitions, a type III super fluid, a pion condensation [18] or another physical phenomena. In such systems, the radial pressure is different from the tangential pressure. This generalization has been very used in the study of the balance and collapse of compact spheres [19-22].

Many researchers have used a great variety of mathematical techniques to try to obtain exact solutions for 
quark stars within the framework of MIT bag model, since it has been demonstrated by Komathiraj and Maharaj [13], Maharaj et al. [23], Sunzu et al. [24], Malaver [25], Thirukkanesh and Maharaj [26] and Thirukkanesh and Ragel [27]. Feroze and Siddiqui [28] and Malaver [29] consider a quadratic equation of state for the matter distribution and specify particular forms for the gravitational potential and electric field intensity. Mafa Takisa and Maharaj [30] obtained new exact solutions to the Einstein-Maxwell system of equations with a polytropic equation of state. Thirukkanesh and Ragel [31] have obtained particular models of anisotropic fluids with polytropic equation of state which are consistent with the reported experimental observations. More recently, Malaver [31,32] generated new exact solutions to the Einstein-Maxwell system considering Van der Waals modified equation of state with and without polytropical exponent. Maharaj et al. [23] found new models by specifying a particular form for one of the gravitational potentials and the measure of anisotropy. Mak and Harko [33] found a relativistic model of strange quark star with the suppositions of spherical symmetry and conformal Killing vector.

Using the procedure suggested by Komathiraj and Maharaj [13] and Maharaj et al. [23] in the present work, we have generated a new class for charged anisotropic matter with the bag equation of state in static spherically symmetric spacetime and a gravitational potential $\mathrm{y}(\mathrm{x})$ of Malaver $[25,34]$ that depends on an adjustable parameter $\alpha$. We have obtained some new classes of static spherically symmetrical models of charged matter considering a particular form for the measure of anisotropy where the variation of the parameter $\alpha$ modifies the radial pressure, tangential pressure, energy density and the electric field intensity of the compact objects. The models of Malaver [25] and Komathiraj and Maharaj [13] for isotropic pressure are recovered as special cases. This article is organized as follows, in Section 2, we present Einstein's field equations. In Section 3 we have particular choices for the gravitational potential $\mathrm{y}(\mathrm{x})$ and the anisotropy that allows solving the field equations and we have obtained new models for charged anisotropic matter. In Section 4, a physical analysis of the new solutions is performed. Finally in Section 5, we conclude.

\section{Einstein Field Equations}

Consider a spherically symmetric four dimensional space time whose line element is given in Schwarzschild coordinates by

$$
d s^{2}=-e^{2 v(r)} d t^{2}+e^{2 \lambda(r)} d r^{2}+r^{2}\left(d \theta^{2}+\sin ^{2} \theta d \varphi^{2}\right)
$$

Using the transformations $x=C r^{2}, Z(x)=e^{-2 \lambda(r)}$ and $A^{2} y^{2}(x)=e^{2 v(r)}$ with arbitrary constants $\mathrm{A}$ and $\mathrm{C}$, suggested by Durgapal and Bannerji [35], the Einstein field equations as given in (1) are

$$
\begin{gathered}
\frac{1-Z}{x}-2 \dot{Z}=\frac{\rho}{C}+\frac{E^{2}}{2 C} \\
4 \mathrm{Z} \frac{\dot{y}}{y}-\frac{1-Z}{x}=\frac{p_{r}}{C}-\frac{E^{2}}{2 C} \\
4 x Z \frac{\ddot{y}}{y}+(4 \mathrm{Z}+2 \mathrm{x} \dot{Z}) \frac{\dot{y}}{y}+\dot{Z}=\frac{p_{t}}{C}+\frac{E^{2}}{2 C} \\
\sigma^{2}=\frac{4 C Z}{x}(x \dot{E}+E)^{2}
\end{gathered}
$$

Where $\rho$ is the energy density, $p_{r}$ is the radial pressure, $E$ is electric field intensity, $\sigma$ is the charge density, $p_{t}$ is the tangential pressure and dot denote differentiation with respect to $\mathrm{x}$.

We can replace the system of fields equations with the bag equation of state by the system

$$
\begin{aligned}
& \rho=3 p+4 B \\
& \frac{p}{C}=Z \frac{\dot{y}}{y}-\frac{1}{2} \dot{Z}-\frac{B}{C} \\
& \frac{E^{2}}{2 C}=\frac{1-Z}{x}-3 Z \frac{\dot{y}}{y}-\frac{1}{2} \dot{Z}-\frac{B}{C} \\
& p_{t}=p_{r}+\Delta \\
& \Delta=\frac{4 x C Z \ddot{y}}{y}+C(2 x \dot{Z}+6 Z) \frac{\dot{y}}{y}+C\left[2\left(\dot{Z}+\frac{B}{C}\right)+\frac{Z-1}{x}\right] \\
& \sigma=2 \sqrt{\frac{C Z}{x}}(E+x \dot{E})
\end{aligned}
$$

The equations (6), (7), (8), (9), (10) and (11) governs the gravitational behavior of a charged quark star. The quantity $\Delta=p_{t}-p_{r}$ is called the measure of anisotropy.

\section{New Exact Solutions with Anisotropy}

Using the method suggested by Komathiraj and Maharaj [13], it is possible to obtain a exact solution of the EinsteinMaxwell system. In this paper, motivated by Malaver [25], we take the particular form of the gravitational potential

$$
y=(a+\alpha x)^{2}
$$

where a is a real constant and $\alpha$ is an adjustable parameter. This potential is regular at the origin and well behaved in the interior of the sphere. Substitution of (12) in (10) allows to obtain the equation of the first order 
$\dot{Z}+\frac{21 x^{2} \alpha^{2}+14 x \alpha a+a^{2}}{8 x^{2} a \alpha+6 x^{3} \alpha^{2}+2 x a^{2}} Z=\frac{\left(1-\frac{2 B}{C} x\right)\left(a^{2}+2 \alpha a x+\alpha^{2} x^{2}\right)+\Delta}{8 x^{2} a \alpha+6 x^{3} \alpha^{2}+2 x a^{2}}$

We specify the measure of anisotropy of the following form:

$$
\Delta=x^{\alpha}+1
$$

$\alpha$ is the adjustable parameter.

$$
Z(x)=\frac{1}{(x+2 a)^{2}(3 x+2 a)}\left[\begin{array}{l}
(x+4 a) C \sqrt{x}-\frac{2 B}{9} x^{4}-\frac{(12 a B-C) x^{3}}{7}-\left(24 a^{2} B-6 a C\right) x^{2}-\frac{4}{3}\left(4 B a^{3}-3 a^{2} C-C\right) x \\
+8 a^{3} C+8 a C
\end{array}\right]
$$

$\mathrm{Z}(\mathrm{x})$ allows generate the following analytical model:

$$
\begin{aligned}
& e^{2 v}=A^{2}\left(a+\frac{1}{2} x\right)^{4} \\
& e^{2 \lambda}=\frac{C(x+2 a)^{2}(3 x+2 a)}{\left[\begin{array}{l}
(x+4 a) C \sqrt{x}-\frac{2 B}{9} x^{4}-\frac{(12 a B-C)}{7} x^{3}-\left(24 a^{2} B-6 a C\right) x^{2}-\frac{4}{3}\left(4 B a^{3}-3 a^{2} C-C\right) x \\
8 a^{3} C+8 a C
\end{array}\right]} \\
& p_{r}=\frac{1}{(x+2 a)^{2}(3 x+2 a)\left(a+\frac{1}{2} x\right)}\left[\begin{array}{l}
(x+4 a) C \sqrt{x}-\frac{2 B}{9} x^{4}-\frac{(12 a B-C)}{7} x^{3}-\left(24 a^{2} B-6 a C\right) x^{2} \\
-\frac{1}{3}\left(16 B a^{3}-12 a^{2} C-4 C\right) x+8 a^{3} C+8 a C
\end{array}\right] \\
& -\frac{1}{2(x+2 a)^{2}(3 x+2 a)}\left[\begin{array}{l}
C \sqrt{x}+\frac{(x+4 a)}{2 \sqrt{x}} C-\frac{8}{9} B x^{3}-\frac{(36 a B-3 C)}{7} x^{2}-\left(48 a^{2} B-12 a C\right) x-\frac{16}{3} B a^{3} \\
+4 a^{2} C+\frac{4}{3} C
\end{array}\right] \\
& +\frac{1}{(x+2 a)^{2}(3 x+2 a)^{2}}\left[\begin{array}{l}
9 C x^{2} \sqrt{x}+44 a C x \sqrt{x}-11 B x^{5}-\frac{5242}{63} a B x^{4}-\frac{2896}{7} a^{2} B x^{3}-480 a^{3} B x^{2}-\frac{560}{3} a^{4} B x \\
+\frac{9}{7} C x^{4}+\frac{386}{7} a C x^{3}+84 a^{2} C x^{2}+12 C x^{2}+104 a^{3} C x+\frac{248}{3} a C x+32 a^{2} C \sqrt{x}+64 a^{4} C \\
+64 a^{2} C
\end{array}\right] \\
& p_{t}=\frac{1}{(x+2 a)^{2}(3 x+2 a)\left(a+\frac{1}{2} x\right)}\left[\begin{array}{l}
(x+4 a) C \sqrt{x}-\frac{2 B}{9} x^{4}-\frac{(12 a B-C)}{7} x^{3}-\left(24 a^{2} B-6 a C\right) x^{2} \\
-\frac{1}{3}\left(16 B a^{3}-12 a^{2} C-4 C\right) x+8 a^{3} C+8 a C
\end{array}\right] \\
& -\frac{1}{2(x+2 a)^{2}(3 x+2 a)}\left[\begin{array}{l}
C \sqrt{x}+\frac{(x+4 a)}{2 \sqrt{x}} C-\frac{8}{9} B x^{3}-\frac{(36 a B-3 C)}{7} x^{2}-\left(48 a^{2} B-12 a C\right) x-\frac{16}{3} B a^{3} \\
+4 a^{2} C+\frac{4}{3} C
\end{array}\right] \\
& +\frac{1}{(x+2 a)^{2}(3 x+2 a)^{2}}\left[\begin{array}{l}
9 C x^{2} \sqrt{x}+44 a C x \sqrt{x}-11 B x^{5}-\frac{5242}{63} a B x^{4}-\frac{2896}{7} a^{2} B x^{3}-480 a^{3} B x^{2}-\frac{560}{3} a^{4} B x \\
+\frac{9}{7} C x^{4}+\frac{386}{7} a C x^{3}+84 a^{2} C x^{2}+12 C x^{2}+104 a^{3} C x+\frac{248}{3} a C x+32 a^{2} C \sqrt{x}+64 a^{4} C \\
+64 a^{2} C+9 x^{4} \sqrt{x}+48 x^{3} \sqrt{x}+88 a^{2} x^{2} \sqrt{x}+64 x \sqrt{x} a^{3}+16 a^{4} \sqrt{x}+9 x^{4}+48 a x^{3}+88 a^{2} x^{2} \\
+64 a^{3} x+16 a^{4}
\end{array}\right]
\end{aligned}
$$




$$
\begin{aligned}
& \rho=\frac{1}{(x+2 a)^{2}(3 x+2 a)\left(a+\frac{1}{2} x\right)}\left[\begin{array}{l}
3(x+4 a) C \sqrt{x}-\frac{2 B}{3} x^{4}-\frac{3(12 a B-C)}{7} x^{3}-\left(72 a^{2} B-18 a C\right) x^{2} \\
-\left(16 B a^{3}-12 a^{2} C-4 C\right) x+24 a^{3} C+24 a C
\end{array}\right] \\
& -\frac{1}{2(x+2 a)^{2}(3 x+2 a)}\left[\begin{array}{l}
3 C \sqrt{x}+\frac{3(x+4 a)}{2 \sqrt{x}} C-\frac{8}{3} B x^{3}-\frac{3(36 a B-3 C)}{7} x^{2}-\left(144 a^{2} B-36 a C\right) x-16 B a^{3} \\
+12 a^{2} C+4 C
\end{array}\right] \\
& +\frac{1}{(x+2 a)^{2}(3 x+2 a)^{2}}\left[\begin{array}{l}
27 C x^{2} \sqrt{x}+132 a C x \sqrt{x}-33 B x^{5}-\frac{5242}{21} a B x^{4}-\frac{8688}{7} a^{2} B x^{3}-1440 a^{3} B x^{2}-560 a^{4} B x \\
+\frac{27}{7} C x^{4}+\frac{1158}{7} a C x^{3}+252 a^{2} C x^{2}+36 C x^{2}+312 a^{3} C x+248 a C x+96 a^{2} C \sqrt{x}+192 a^{4} C \\
+192 a^{2} C+36 B x^{4}+192 a B x^{3}+352 a^{2} B x^{2}+256 a^{3} B x+64 B a^{4}
\end{array}\right] \\
& E^{2}=\frac{1}{x(x+2 a)^{2}(3 x+2 a)}\left[\begin{array}{l}
6 C x^{3}+28 a C x^{2}+40 a^{2} C x-2(x+4 a) C \sqrt{x}+\frac{4}{9} B x^{4}+\frac{(24 a B-2 C)}{7} x^{3} \\
\left(48 a^{2} B-12 a C\right) x^{2}+\frac{8}{3}\left(4 B a^{3}-3 a^{2} C-C\right) x-8 a C
\end{array}\right] \\
& -\frac{1}{C(x+2 a)^{2}(3 x+a)\left(a+\frac{1}{2} x\right)}\left[\begin{array}{l}
6(x+4 a) C \sqrt{x}-\frac{4}{3} B x^{3}-\frac{(72 a B-6 C)}{7} x^{3}-\left(144 a^{2} B-36 a C\right) x^{2} \\
-\left(32 B a^{3}-24 a^{2}-8 C\right) x+48 a^{3} C+48 a C
\end{array}\right] \\
& -\frac{1}{(x+2 a)^{2}(3 x+2 a)}\left[\begin{array}{l}
C \sqrt{x}+\frac{(x+4 a) C}{2 \sqrt{x}}+\frac{46}{9} B x^{3}+\frac{3}{7}\left(\frac{160}{3} a B+C\right) x^{2}-4\left(2 a^{2} B-3 a C\right) x+\frac{32}{3} B a^{3} \\
+4 a^{2} C+\frac{4}{3} C
\end{array}\right] \\
& +\frac{1}{(x+2 a)^{3}(3 x+2 a)^{2}}\left[\begin{array}{l}
9 C x^{2} \sqrt{x}+44 a C x \sqrt{x}-2 B x^{5}-\frac{1084}{63} a B x^{4}+\frac{9}{7} C x^{4}-\frac{1608}{7} a^{2} B x^{3}+\frac{386}{7} a C x^{3} \\
-240 B a^{3} x^{2}+84 a^{2} C x^{2}+12 C x^{2}+104 a^{3} C x+\frac{248}{3} a C x+32 C a^{2} \sqrt{x}-\frac{128}{3} B a^{4} x \\
+64 a^{4} C+64 a^{2} C
\end{array}\right]
\end{aligned}
$$

The metric for this model is

$$
d s^{2}=-A^{2}\left(a+\frac{1}{2} x\right)^{4} d t^{2}+\left[\frac{C(x+2 a)^{2}(3 x+2 a)}{4 c x\left(\begin{array}{l}
(x+4 a) C \sqrt{x}-\frac{2}{9} B x^{4}-\frac{(12 a B-C)}{7} x^{3}-6\left(4 a^{2} B-a C\right) x^{2} \\
-\frac{4}{3}\left(4 B a^{3}-3 a^{2} C-C\right) x+8 a^{3} C+8 a C
\end{array}\right.}\right] d x^{2}+\frac{x}{c}\left(d \theta^{2}+\sin ^{2} \theta d \varphi^{2}\right)
$$

With $\alpha=1$, the eq. (13) becomes

$$
\dot{Z}+\frac{21 x^{2}+14 x a+a^{2}}{8 x^{2} a+6 x^{3}+2 x a^{2}} Z=\frac{\left(1-\frac{2 B}{C} x\right)\left(a^{2}+2 a x+x^{2}\right)+x+1}{8 x^{2} a+6 x^{3}+2 x a^{2}}
$$

Integrating (24), we obtain 


$$
Z(x)=\frac{1}{315(x+a)^{2}(3 x+a)}\left[\begin{array}{l}
3\left(15 x^{3}+63 a x^{2}+21 x^{2}+105 a^{2} x+35 x+35 a x+105 a^{3}+105 a\right) \\
-\frac{2 B x}{C}\left(35 x^{3}+135 a x^{2}+189 a^{2} x+105 a^{3}\right)
\end{array}\right]
$$

With the equation (25), we can generate the exact analytical model

$$
\begin{aligned}
& e^{2 v}=A^{2}(a+x)^{4} \\
& e^{2 \lambda}=\frac{315(x+a)^{2}(3 x+a)}{\left[3\left(15 x^{3}+63 a x^{2}+21 x^{2}+105 a^{2} x+35 x+35 a x+105 a^{3}+105 a\right)\right.} \\
& \left.-\frac{2 B x}{C}\left(35 x^{3}+135 a x^{2}+189 a^{2} x+105 a^{3}\right)\right] \\
& p_{r}=\frac{C}{315(x+a)^{3}(3 x+a)}\left[\begin{array}{l}
6\left(15 x^{3}+63 a x^{2}+21 x^{2}+105 a^{2} x+35 x+35 a x+105 a^{3}+105 a\right) \\
-\frac{4 B x}{C}\left(35 x^{3}+135 a x^{2}+189 a^{2} x+105 a^{3}\right)
\end{array}\right] \\
& +\frac{1}{630(x+a)^{2}(3 x+a)}\left[\begin{array}{l}
280 B x^{3}-135 C x^{2}+810 a B x^{2}+756 a^{2} B x-378 a C x-126 C x-315 a^{2} C \\
-105 C+210 a^{3} B-105 a C
\end{array}\right] \\
& -\frac{1}{315(x+a)^{3}(3 x+a)}\left[\begin{array}{l}
1015 B x^{4}+3420 a B x^{3}+4158 a^{2} B x^{3}+2100 a^{3} B x-45 C x^{3}-189 a C x^{2}-63 C x^{2} \\
-315 a^{2} C x-105 C x-105 a C x-315 a^{3} C-315 a C+315 B a^{4}
\end{array}\right] \\
& -\frac{1}{210(x+a)^{2}(3 x+a)^{2}}\left[\begin{array}{l}
70 B x^{4}-45 C x^{3}+270 a B x^{3}+378 a^{2} B x^{2}-189 a C x^{2}-63 C x^{2}-315 a^{2} C x-105 C x \\
+210 a^{3} B x-105 a C x-315 C a^{3}-315 a C
\end{array}\right] \\
& p_{t}=\frac{C}{315(x+a)^{3}(3 x+a)}\left[\begin{array}{l}
6\left(15 x^{3}+63 a x^{2}+21 x^{2}+105 a^{2} x+35 x+35 a x+105 a^{3}+105 a\right) \\
-\frac{4 B x}{C}\left(35 x^{3}+135 a x^{2}+189 a^{2} x+105 a^{3}\right)
\end{array}\right] \\
& +\frac{1}{630(x+a)^{2}(3 x+a)}\left[\begin{array}{l}
1890 x^{4}+280 B x^{3}-135 C x^{2}+810 a B x^{2}+756 a^{2} B x-378 a C x-126 C x-315 a^{2} C \\
-105 C+210 a^{3} B-105 a C+4410 a x^{3}+3150 a^{2} x^{2}+630 a^{3} x+1890 x^{3}+4410 a x^{2} \\
+3150 a^{2} x+630 a^{3}
\end{array}\right] \\
& -\frac{1}{315(x+a)^{3}(3 x+a)}\left[\begin{array}{l}
1015 B x^{4}+3420 a B x^{3}+4158 a^{2} B x^{3}+2100 a^{3} B x-45 C x^{3}-189 a C x^{2}-63 C x^{2} \\
-315 a^{2} C x-105 C x-105 a C x-315 a^{3} C-315 a C+315 B a^{4}
\end{array}\right] \\
& -\frac{1}{210(x+a)^{2}(3 x+a)^{2}}\left[\begin{array}{l}
70 B x^{4}-45 C x^{3}+270 a B x^{3}+378 a^{2} B x^{2}-189 a C x^{2}-63 C x^{2}-315 a^{2} C x-105 C x \\
+210 a^{3} B x-105 a C x-315 C a^{3}-315 a C
\end{array}\right] \\
& \rho=\frac{3 C}{315(x+a)^{3}(3 x+a)}\left[\begin{array}{l}
6\left(15 x^{3}+63 a x^{2}+21 x^{2}+105 a^{2} x+35 x+35 a x+105 a^{3}+105 a\right) \\
-\frac{4 B x}{C}\left(35 x^{3}+135 a x^{2}+189 a^{2} x+105 a^{3}\right)
\end{array}\right] \\
& +\frac{1}{630(x+a)^{2}(3 x+a)}\left[\begin{array}{l}
8400 B x^{3}-405 C x^{2}+20070 a B x^{2}+14868 a^{2} B x-1134 a C x-378 C x-955 a^{2} C \\
-315 C+3150 a^{3} B-315 a C
\end{array}\right] \\
& -\frac{3}{315(x+a)^{3}(3 x+a)}\left[\begin{array}{l}
1015 B x^{4}+3420 a B x^{3}+4158 a^{2} B x^{3}+2100 a^{3} B x-45 C x^{3}-189 a C x^{2}-63 C x^{2} \\
-315 a^{2} C x-105 C x-105 a C x-315 a^{3} C-315 a C+315 B a^{4}
\end{array}\right] \\
& -\frac{3}{210(x+a)^{2}(3 x+a)^{2}}\left[\begin{array}{l}
70 B x^{4}-45 C x^{3}+270 a B x^{3}+378 a^{2} B x^{2}-189 a C x^{2}-63 C x^{2}-315 a^{2} C x-105 C x \\
+210 a^{3} B x-105 a C x-315 C a^{3}-315 a C
\end{array}\right]
\end{aligned}
$$




$$
\begin{aligned}
& E^{2}=\frac{2 C}{315 x(x+a)^{2}(3 x+a)}\left[\begin{array}{l}
900 x^{3}-2016 a x^{2}+1260 a^{2} x-63 x^{2}-105 x-105 a x-945 a \\
-\frac{2 B x}{C}\left(35 x^{3}+135 a x^{2}+189 a^{2} x+105 a^{3}\right)
\end{array}\right] \\
& -\frac{2 C}{315(x+a)^{3}(3 x+a)}\left[\begin{array}{l}
270 x^{3}+378 x^{2}+1134 a x^{2}+1890 a^{2} x+630 x+630 a x+1890 a^{3}+5670 a \\
-\frac{2 B x}{C}\left(210 x^{3}+810 a x^{2}+1134 a^{2} x+630 a^{3}\right)
\end{array}\right] \\
& -\frac{2}{315(x+a)^{3}(3 x+a)}\left[\begin{array}{l}
1015 B x^{4}+3420 a B x^{3}+4158 a^{2} B x^{2}+2100 a^{3} B x-45 C x^{3}-189 a C x^{2}-63 C x^{2}-315 a^{2} C \\
-105 C x-105 a C x-315 C a^{3}-315 a C+315 B a^{4}
\end{array}\right] \\
& -\frac{1}{315(x+a)^{2}(3 x+a)}\left[\begin{array}{l}
280 a B x^{3}-135 C x^{2}+810 a B x^{2}+756 a^{2} B x-378 a C x-126 C x-315 C a^{2}-105 C+210 B a^{3} \\
-105 a C
\end{array}\right] \\
& -\frac{1}{105(x+a)^{2}(3 x+a)^{2}}\left[\begin{array}{l}
70 B x^{4}-45 C x^{3}+270 a B x^{3}+378 a^{2} B x^{2}-189 a C x^{2}-63 C x^{2}-315 a^{2} C x-105 C x+210 a^{3} B x \\
-105 a C x-315 C a^{3}-315 a C
\end{array}\right]
\end{aligned}
$$

The metric for this model is

$$
d s^{2}=-A^{2}(a+x)^{4} d t^{2}+\left[\frac{315(x+a)^{2}(3 x+a)}{4 c x\left(\begin{array}{l}
3\left(15 x^{3}+63 a x^{2}+21 x^{2}+105 a^{2} x+35 x+35 a x\right. \\
\left.+105 a^{3}+105 a\right)-\frac{2 B x}{C}\left(35 x^{3}+135 a x^{2}+189 a^{2} x+105 a^{3}\right)
\end{array}\right)}\right] d x^{2}+\frac{x}{c}\left(d \theta^{2}+\sin ^{2} \theta d \phi^{2}\right)
$$

\section{Physical Analysis}

The presented models constitute another new family of solutions for a charged quark star with anisotropic pressure. The metric functions can be written in terms of polinominal functions, and the variables energy density, pressure and charge density also are represented analytical. For the case $\alpha=1 / 2$, The metric functions $e^{2(v(r)}$ and $e^{2 \lambda(r)}$ behaves well inside the star and have a finite value of $e^{2 x(r)}=a^{4} A^{2}$ and $e^{22(r)}=\frac{a^{2}}{a^{2}+1}$ in $\mathrm{x}=0$ but in the energy density, radial pressure, tangential pressure and the electrical field intensity admits singularity at the center $\mathrm{x}=0$ as in the quark star model of Sunzu et al. [24]. When $\Delta=0$, we obtain the Malaver model [25] for isotropic pressure as a special case.

For the solution found with $\alpha=1$, the functions $e^{2(r)}$ and $e^{2(x)}$ acquire finite values in the center $\mathrm{x}=0$ as in the case for $\alpha=1 / 2$. The energy density, the radial pressure and the tangential pressure at the center take the values $\rho=\frac{2642 a^{2} C+13 C-a C}{2 a^{3}}+2 B \quad, \quad p_{r}=\frac{27 a^{2} C-a C+27 C}{6 a^{3}}-\frac{2 B}{3}$, $p_{t}=\frac{24 a^{2} C+16 c+6 a^{3}}{6 a^{3}}-\frac{2 B}{3}$, respectively. Again, there is a singularity in the electrical field as in the quark star model of Malaver [34] . For isotropic pressure $\Delta=0$ and we recover the Komathiraj and Maharaj model [13] with $\alpha=1$ as a especial case.

\section{Conclusion}

We have generated a new class of exact solutions for the Einstein-Maxwell system within the framework of MIT Bag Model with a particular form for the measure of anisotropy. We have studied two new types of analytical solutions specifying the form of the gravitational potential $y(x)$ which depends on an adjustable parameter $\alpha$. The first solution admits a singularity in the radial pressure, tangential pressure, energy density and electric field at the center of the stellar object and the second solution is regular throughout the interior but present a singularity in the electric field intensity. The proposed model allows us to recover the solutions with isotropic pressures as a special case. The method to generate analytical models with anisotropy will depend on the form of and $y(x)$ and $\Delta$, necessary to determine physically acceptable solutions.

\section{References}

[1] Kuhfitting, P.K.(2011). Some remarks on exact wormhole solutions, Adv. Stud. Theor. Phys., 5, 365- 367.

[2] Bicak, J.(2006). Einstein equations: exact solutions, Encyclopedia of Mathematical Physics, 2, 165-173.

[3] Malaver, M. (2013). Black Holes, Wormholes and Dark Energy Stars in General Relativity. Lambert Academic Publishing, Berlin. ISBN: 978-3-659-34784-9. 
[4] Komathiraj, K., and Maharaj,S.D. (2008). Classes of exact Einstein-Maxwell solutions, Gen. Rel. Grav., 39, 2079-2093.

[5] Sharma, R., Mukherjee, S and Maharaj, S.D.(2001). General solution for a class of static charged stars, Gen.Rel. Grav., 33, 999-110.

[6] Dey, M, Bombaci, I, Dey, J, Ray, S and Samantra, B.C. (1998) Phys. Lett. B438,123.

[7] Itoh,N.(1970). Prog. Theor. Phys.44, 291.

[8] Schwarzschild, K. (1916). Über das Gravitationsfeld einer Kugel aus inkompressibler Flüssigkeit, Math. Phys. Tech, 424-434.

[9] Tolman, R.C. (1939). Static Solutions of Einstein's Field Equations for Spheres of Fluid, Phys. Rev., 55, 364-373.

[10] Oppenheimer, J.R. and Volkoff, G. (1939). On massive neutron cores, Phys. Rev., 55, 374- 381.

[11] Chandrasekhar, S. (1931). Mass of Ideal White Dwarfs, Astrophys. J., 74, 81-82.

[12] Baade, W., and Zwicky, F. (1934). Cosmic Rays from SuperNovae, Proc. Nat. Acad. Sci. U. S., (20), 259-263.

[13] Komathiraj, K., and Maharaj, S.D.(2007). Analytical models for quark stars, Int.J.Mod. Phys., D16, pp. 1803-1811.

[14] Herrera, L., and Santos, N.O. (1997), Phys. Rep.286, 53.

[15] Cosenza, M., Herrera, L., Esculpi, M. and Witten, L.(1981), J.Math.Phys., 22(1), 118.

[16] Gokhroo, M.K., and Mehra. A.L. (1994). Anisotropic spheres with variable energy density in general relativity, Gen.Relat.Grav., 26(1), 75-84.

[17] Herrera, L. (1992), Phys.Lett., A165, 206.

[18] Herrera, L. (1992), Phys.Lett., A165, 206.

[19] Herrera, L., Ruggeri, G.J and Witten. L. (1979),Astrophys. J., 234, 1094.

[20] Herrera, L., and Ponce de Leon. J. (1985), J. Math.Phys., 26, 2018.

[21] Herrera, L., and Santos. N.O. (1998), J. Math. Phys., 39, 3817.

[22] Bondi.H.(1992), Mon. Not. R. Astron. Soc., 259, 365.
[23] Maharaj, S.D., Sunzu, J.M. and Ray, S. (2014). Eur. Phys. J.Plus., 129, 3.

[24] Sunzu, J.M, Maharaj, S.D and Ray, S.(2014). Quark star model with charged anisotropic matter, accepted for publication in Astrophysics and Space Science, published online September 25, 2014. DOI: 10.1007/s10509-0142131-4.

[25] Malaver, M. (2009). Análisis comparativo de algunos modelos analíticos para estrellas de quarks, Revista Integración, 27, 125-133.

[26] Thirukkanesh, S., and Maharaj, S.D. (2008). Charged anisotropic matter with linear equation of state, Class. Quantum Gravity, 25, 235001.

[27] Thirukkanesh, S., and Ragel, F.C. (2013). A class of exact strange quark star model, PRAMANA-Journal of physics, 81(2), 275-286.

[28] Feroze, T. and Siddiqui, A. (2011). Charged anisotropic matter with quadratic equation of state, Gen. Rel. Grav., 43, 10251035 .

[29] Malaver, M. (2014). Strange Quark Star Model with Quadratic Equation of State, Frontiers of Mathematics and Its Applications., 1(1), 9-15.

[30] Takisa, P.M., and Maharaj, S.D. (2013). Some charged polytropic models, Gen.Rel.Grav., 45, 1951-1969.

[31] Malaver, M. (2013). Analytical model for charged polytropic stars with Van der Waals Modified Equation of State, American Journal of Astronomy and Astrophysics, 1(4), 41-46.

[32] Malaver, M. (2013). Regular model for a quark star with Van der Waals modified equation of state, World Applied Programming., 3, 309-313.

[33] Mak, M.K., and Harko, T. (2004). Quark stars admitting a one-parameter group of conformal motions, Int.J.Mod.Phys, D13, 149-156.

[34] Malaver, M. (2014). Models for Quark Stars with Charged Anisotropic Matter, Research Journal of Modeling and Simulation, 1(4), 65-71.

[35] Durgapal, M.C., and Bannerji, R. (1983). New analytical stellar model in general relativity, Phys.Rev. D27, 328-331. 